\title{
Semantic Segmentation in Aerial Images Using Class-Aware Unsupervised Domain Adaptation
}

\author{
Ying Chen, Xu Ouyang, Kaiyue Zhu, Gady Agam \\ ychen245,xouyang3,kzhu6@hawk.iit.edu,agam@iit.edu \\ Illinois Institute of Technology \\ Chicago, Illinois, United States
}

\begin{abstract}
Semantic segmentation using deep neural networks is an important component of aerial image understanding. However, models trained using data from one domain may not generalize well to another domain due to a domain shift between data distributions in the two domains. Such a domain gap is common in aerial images due to large visual appearance changes, and so substantial accuracy loss may occur when using a trained model for inference on new data. In this paper, we propose a novel unsupervised domain adaptation framework to address domain shift in the context of semantic segmentation of aerial images. To this end, we address the problem of domain shift by learning class-aware distribution differences between the source and target domains. Further, we employ entropy minimization on the target domain to produce high-confidence predictions. We demonstrate the effectiveness of the proposed approach using a challenge segmentation dataset by ISPRS, and show improvement over state-of-the-art methods.
\end{abstract}

\section{CCS CONCEPTS}

\section{- Computing methodologies $\rightarrow$ Image segmentation.}

\section{KEYWORDS}

Aerial image understanding, Domain adaptation, Semantic segmentation, Class-wise distribution

ACM Reference Format:

Ying Chen, Xu Ouyang, Kaiyue Zhu, Gady Agam. 2021. Semantic Segmentation in Aerial Images Using Class-Aware Unsupervised Domain Adaptation. In 4th ACM SIGSPATIAL International Workshop on AI for Geographic Knowledge Discovery (GEOAI '21), November 2-5, 2021, Beijing, China. ACM, New York, NY, USA, 8 pages. https://doi.org/10.1145/3486635.3491069

\section{INTRODUCTION}

Semantic segmentation is a core image analysis operation where each pixel is assigned a semantic label attributing it to a certain object. In practice, semantic segmentation normally serves as highlevel processing step to support a complete scene understanding. In the context of remote sensing[7], semantic segmentation is viewed as pixel-wise classification and has been widely studied in a variety of potential applications and tasks concerning aerial images, such

This work is licensed under a Creative Commons Attribution International 4.0 License. GEOAI '21, November 2-5, 2021, Beijing, China

(C) 2021 Copyright held by the owner/author(s)

ACM ISBN 978-1-4503-9120-7/21/11.

https://doi.org/10.1145/3486635.3491069 as monitoring and planning urban areas, disaster detection, and fast emergency response. The growing use of Unmanned Aerial Vehicles (UAVs)[11] increase the need for semantic segmentation of high resolution images to support automatic monitoring of inhabited areas.

CNN-based fully-supervised approaches $[4,28]$ have achieved remarkable progress in semantic segmentation on datasets such as Cityscapes [10] and PASCAL VOC-2012 [12]. In practice, given sufficient labeled data, training a state-of-the-art network can easily achieve an accuracy over $80 \%$ [24]. However, the high efficiency of deep models relies on a robust labeled dataset.

Producing dense pixel-level annotations for real-world datasets is extremely costly and requires an enormous amount of manual work. For example, the annotation of a single Cityscapes image takes nearly 90 minutes on average [33]. Using a small training set results in poor generalization from training/source data to test/target data when samples of the target domain have different characteristics. One straightforward way to address this challenge is to apply weakly and semi-supervised methods [37, 42], where only a small subset of the data is annotated, or where only weak supervision is provided. The application of such approaches may still require timeconsuming labeling and weak annotation may be hard to obtain for most real-world applications. Thus, there is a need for efficient ways to address semi-supervised or unsupervised domain adaptation that requires a small subset of target domain labeled data or even no target domain labeled data. In this work, we focus on unsupervised domain adaptation.

Unsupervised domain adaptation (UDA) aims at learning a target domain model from a well performing source model. UDA tends to reduce or remove cross-domain shift between the source and target domains by learning a mapping function between them. Most recent UDA methods address this cross-domain shift through minimizing the distribution difference between the intermediate features or the final outputs of the two domains. Some approaches [29, 30, 45] use maximum mean discrepancies (MMD) or adversarial training $[19,39-41]$ to solve this issue, while other methods apply self-training $[26,48]$ to produce pseudo labels, or apply generative networks $[1,2,18,43]$ to generate target data. Semi-supervised learning solves a problem related to domain adaptation and many of its strategies can be used to address domain adaptation, such as self-training, and entropy minimization [26, 41].

Applying entropy minimization to the target domain is helpful to avoid over-confident source domain decisions. However, there may still be a distribution difference between the source and target domains such as appearance differences between them. Significant appearance differences between source and target domains are common in aerial images (e.g. buildings, roads, trees), and so existing 


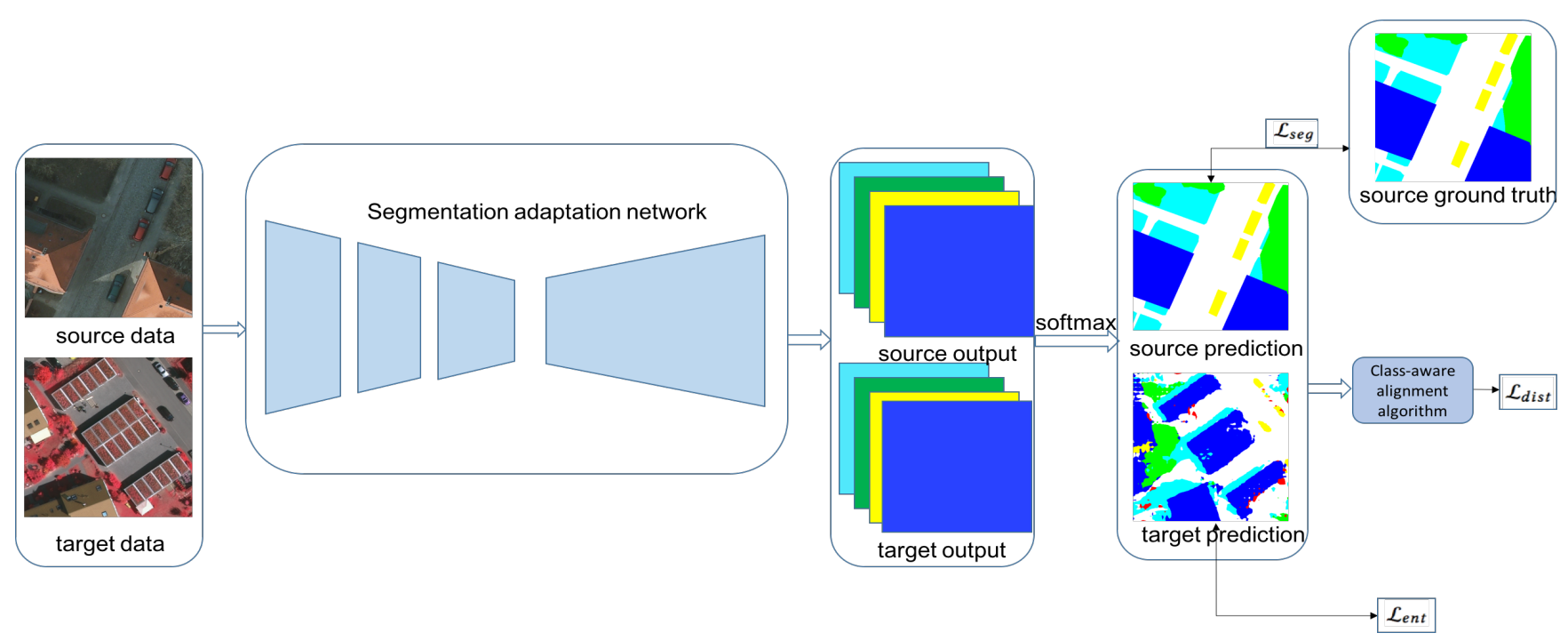

Figure 1: Proposed network architecture and loss. $\mathcal{L}_{\text {ent }}$ minimizes the entropy of the target sample $x_{t}$. While $\mathcal{L}_{d i s t}$ is used to enforce the class-wise distribution consistency between the source and target domains.

methods may not perform well for such problems. We propose to to approach both issues by applying entropy minimization as well as addressing distribution difference between the source and target domains.

In this work, we propose a new framework for domain adaptation of semantic segmentation in aerial images using a neural network. An overview of the proposed network is shown in Figure1. Our approach involves two strategies: First, we employ entropy minimization to UDA following an observation that models trained on labeled source domain and unlabeled target domain tend to get over-confident/low-entropy and under-confident/high-entropy results respectively [41]. Thus, to address this we use entropy minimization on the target domain to reduce its entropy. Second, to reduce the domain shift between the source and target domains we learn a class-aware distribution difference between the source and target domains instead of attempting to align pixel-level feature distributions. The source and target domain have different class distributions (for example, the source domain has more buildings compared to the target domain), and our goal is to do domain adaptation while taking into account this distribution difference, which is termed as class-aware distribution difference. We use the Kullback-Leibler divergence (KL-divergence) [22] to address the class-wise distribution difference between the source and target domains. To the best of our knowledge, we are the first to apply class-wise distribution alignment to address domain adaptation in semantic segmentation.

The key contributions of this paper are as follows:

- We propose a simple yet effective end-to-end approach. In contrast to common methods for domain adaptation which are adversarial and are known to be difficult to train [27], the proposed method is not adversarial and involves a backbone network which requires a smaller number of parameters for training.
- We propose learning class-aware distributions of the source and target domains to address appearance differences between them, and demonstrate the usefulness of this approach for unsupervised domain adaptation (UDA) in satellite images.

- We apply entropy minimization to improve low-confidence predictions on the target domain for semantic segmentation of aerial images.

- We demonstrate that our approach is able to mitigate domain shift between source and target domains and surpass stateof-the-art methods when evaluated on the standard ISPRS segmentation challenge dataset.

\section{RELATED WORK}

This section summarizes related work for domain adaptation in semantic segmentation.

\subsection{Semantic Segmentation}

In the past decade, numerous semantic segmentation methods based on deep neural networks (e.g. FCN [28], DeepLab [4]) have been developed and trained using real-world datasets (e.g. Cityscapes [10]). Such networks use context information and high-level representation that are learned by deep stacked layers. The state-of-the-art performance for such networks depends on having large amounts of high-quality annotated data, and may not generalize well to unseen test data from a different domain. One approach for addressing this issue is to adopt the networks using weakly or semi-supervised approaches [20, 37, 42]. In this way, only a small set of dense annotated data or weak annotation (e.g. bounding box instead of a pixel map) is required. Another possible approach for addressing the issue is to employ synthetic datasets. Synthetic images can be generated based on virtual worlds and can be produced with dense annotation automatically. Training using synthetics images, however, may result in a poor performance on actual data due to 
appearance difference between synthetic images and real-world images.

\subsection{Unsupervised Domain Adaptation}

Domain adaptation aims at helping a trained model better generalize to unseen test data. Numerous unsupervised domain adaptation (UDA) methods [29-31, 36, 44] for image classification and detection have been proposed to address the domain shift between labeled source and unlabeled target domains. The main idea behind current approaches is to minimize the distribution discrepancy between source and target data. The main approaches for addressing such distribution discrepancy can be classified as global distribution alignment (i.e. aligning the marginal distributions of samples between the source and target domains), and class-wise conditional distribution alignment (i.e. aligning the distributions conditioned on labels between the source and target domains as in conditional GAN). Recent work include maximum mean discrepancies (MMD) [29, 30], adversarial training [39-41], and self-training with pseudo labels $[26,48]$. Most of existing UDA methods are adversarial.

\subsection{Non-Generative UDA for Semantic Segmentation}

Most of the recent work concerning UDA for semantic segmentation use synthetic data as the source domain (e.g. SYNTHIA [34]). Adversarial training is the most common method for UDA on semantic segmentation. It uses two networks. One network aims to predict the segmentation map for labeled training data from either the source or target domain, while the other network is used to align feature distribution between the two domains taken from the segmentation network in an adversarial manner. The first unsupervised domain adaptation for transferring semantic segmentation FCNs across image domains was proposed by Hoffman et al. [19], where they use global and category specific adaptation techniques to combine global and local alignment methods. Chen et al. [8] propose a similar approach to perform global and class-wise adaptation, and use adversarial learning for assigning pseudo labels to achieve joint global and class-wise adaptation of segments.

In [6] adversarial training is used to adapt from synthetic to real urban scenes by using spatial-aware adaptation loss along with a distillation loss. In this approach, feature maps are divided into multiple grids and a MMD loss is calculated in each grid cell. Hong et al. [21] propose a principled approach to model the residual in the feature space between the source and target domains while maintaining their semantic spatial layouts. In [39], Tsai et al. propose an adversarial learning approach in output space to benefit from spatial similarities between the source and target domains. In addition, they perform domain adaptation at different feature levels. $\mathrm{Vu}$ et al. [41] propose an adversarial entropy minimization approach to address domain shift between source and target domains with losses based on the entropy of pixel-wise predictions. Their work involves two complementary methods using an entropy loss and an adversarial loss respectively. Considering that the gradient of the entropy is biased towards samples that are easy to transfer, a similar work is proposed in [5] where a maximum squares loss is used to replace the entropy loss.

\subsection{Generative UDA for Semantic Segmentation}

Generative networks are used of domain adaptation on semantic segmentation by conditioning target images on the source domain. In [18], Hoffman et al. design an approach called a Cycle-Consistent Adversarial Domain Adaptation (CyDADA). The model performs both pixel-level adaptation and feature-level representation adaptation. They use CycleGAN [47] to produce a target image conditioned on a source image. To preserve spatial structures and semantic information, $\mathrm{Wu}$ et al. [43] perform channel-wise feature alignment in both an image generator and a segmentation network. The generator produces samples from the target domain with content from source domain while maintaining the style of target domain. In [26], Li et al. propose a bidirectional learning framework to alternate learning image translation using CycleGAN. Similar work in [2] and [1] employ CycleGAN based image translation to translate the target domain to the source domain and increase the ability of the model to work on the new target domain.

\subsection{Self-supervised DA for Semantic Segmentation}

Another category of approaches addressing UDA involves selftraining. The core idea is to generate pseudo labels for unlabeled target domain data using an ensemble of previous models. Zou et al. [48] propose an iterative self-training (ST) procedure to generate pseudo labels for target data and use them to retrain the model. Class balancing is used to avoid gradual dominance of the majority class, and spatial priors are used to refine the generated labels. In [26], self-training is employed to learn a better segmentation model and in return improve an image translation model.

Entropy minimization has been demonstrated to be useful for addressing semi-supervised learning problems [15, 38]. It has also been used to solve domain adaptation for classification [30] and semantic segmentation on natural images [41]. To the best of our knowledge, we are the first to apply entropy based UDA for semantic segmentation in aerial images.

\subsection{Meta-learning DA for Semantic Segmentation}

Meta-learning solves the problem of learning to learn and has been successfully applied to tackle various applications. The principle of meta-learning [17] has also been studied for the domain adaptation problem [9, 14, 25]. Both Chen et al. [9] and Gong et al. [14] employ mata-learning to address the domain adaptation problem when there are multiple unknown target domains. The goal of Zhang et al. [46] and Gonget al. [14] is to improve the domain generalization performance for semantic segmentation model using a Model-agnostic meta-learning (MAML) [13] strategy.

The key limitations of such approaches are two fold: first, some of these approaches are not end-to-end; second, most of these approaches try to learn a common low-level feature representation to address the domain shift, which assumes that similar objects in different domains should have similar semantic appearance. Satellite images from different domains may not share semantic appearance. Our proposed approach is able to address these limitations. 


\section{APPROACH}

In this section, we present the proposed framework of domain adaptation for semantic segmentation in aerial images, where we incorporate two mechanisms of entropy minimization and classwise distribution alignment.

\subsection{Supervised Semantic Segmentation}

Our domain adaptation approach relies on a single segmentation adaptation network. An overview of the proposed model is provided in Figure 1.

The model is first learned on the source domain using a supervised segmentation loss. We denote the labeled source domain set as $D_{s}=\left\{\left(x_{s}, y_{s}\right) \mid x_{s} \in R^{H \times W \times 3}, y_{s} \in(1, C)^{H \times W}\right\}$, where each sample $x_{s}$ is an $H \times W$ color image which is associated with a ground-truth $C$-class segmentation map $y_{s}$. Each entry $y_{s}^{i, j}$ takes a class label from a finite set $(1,2, \ldots, C)$ or a one-hot vector $\left[y_{s}^{(i, j, c)}\right]_{c}$. Similarly, we denote the unlabeled target domain set using $D_{t}=$ $\left\{\left(x_{t}\right) \mid x_{t} \in R^{H \times W \times 3}\right\}$. We forward the source image $x_{s}$ to a semantic segmentation network $F$. The network output is a segmentation map $F\left(x_{s}\right)$ with dimension $H \times W \times C$. After passing through a softmax layer, we predict the segmentation class probability $Q_{x_{s}}=\left[Q_{x_{s}}^{(i, j, c)}\right]_{i, j, c}=\operatorname{softmax}\left(F\left(x_{s}\right)\right)$. The model $F$ is trained on source data in a supervised manner using the categorical cross entropy loss:

$$
\mathcal{L}_{s e g}\left(x_{s}, y_{s}\right)=-\frac{1}{N} \sum_{i=1}^{N} \sum_{c=1}^{C} y_{s}^{(i, c)} \log Q_{x_{s}}^{(i, c)}
$$

where $N=H \times W$ is the total number of pixels in an image.

\subsection{Entropy Minimization}

In our UDA approach, the target domain is unlabeled, and so supervised learning based on the source domain may result in producing over-confident predictions for source examples, and underconfident predictions for target examples.

To improve the test performance on target data, we could use a self-supervised learning (SSL) method to select target pixel predictions with sufficient confidence and use them as pseudo labels for target samples during training. Specifically, based on the prediction probability produced for the target domain, we could use SSL to obtain pseudo labels $\hat{y}_{t}$ with high confidence, using a fixed or scheduled threshold. The categorical cross entropy loss on target predictions using these pseudo labels is given by:

$$
\mathcal{L}_{c e}\left(x_{t}, \hat{y}_{t}\right)=-\frac{1}{N} \sum_{i=1}^{N} \sum_{c=1}^{C} y_{t}^{(i, c)} \log Q_{x_{t}}^{(i, c)}
$$

In contrast to SSL where a threshold needs to be selected for hard assignment, in the proposed approach we use soft assignment. Specifically, we use the entropy minimization method [41] to constrain the model to directly produce high confidence predictions, by minimizing the Shannon entropy [35] of target domain predictions. The entropy loss $\mathcal{L}_{\text {ent }}$ of [41] is given by:

$$
\mathcal{L}_{\text {ent }}\left(x_{t}\right)=-\frac{1}{N} \sum_{i=1}^{N} \frac{1}{\log (C)} \sum_{c=1}^{C} Q_{x_{t}}^{(i, c)} \log Q_{x_{t}}^{(i, c)}
$$

By comparing $\mathcal{L}_{e n t}\left(x_{t}\right)$ and $\mathcal{L}_{c e}\left(x_{t}, \hat{y}_{t}\right)$, we observe that these two loss terms are nearly equivalent except that $\mathcal{L}_{\text {ent }}\left(x_{t}\right)$ is a softassignment version of $\mathcal{L}_{c e}\left(x_{t}, \hat{y}_{t}\right)$. Using a soft assignment in the proposed approach avoids the need for threshold selection in SSL which may be unstable to produce target domain predictions with high-confidence/certainty,

\subsection{Learning Class-aware Distribution Differences}

By applying entropy minimization, the proposed approach is able to produce low-entropy when trained on unlabeled target data. However, there may still be a distribution difference between the source and target domains. To address this, we propose to learn class-aware distribution, which refers to distribution of sample counts in source or target, between different domains. The approach is illustrated in Algorithm 1. The purpose of the class-wise distribution alignment term in the loss function is treated as a regularization term able to address different types of domain differences, which has the same working principle as other methods for addressing domain shift, like GAN-based method aiming to learn a common feature representation between different domains. Our regularization term works as follows: if the class-wise distributions are similar between the two domains, we can use a larger lambda, which controls the strength of the regularization, to enforce the network to learn to reduce the class-wise distribution difference; otherwise, we can use a smaller lambda to help alleviate the domain shift on some level by learning the distribution difference.

We first apply the softmax function to the logits $F\left(x_{s}\right)$ and $F\left(x_{t}\right)$ produced by the network $F$ for source data and target data respectively to generate $P_{x_{s}}$ and $P_{x_{t}}$. We then add all the values of $P_{x_{s}}$ and $P_{x_{t}}$ via the channel dimension to produce C-dimensional vectors, and calculate soft counts for each class in both domains. Finally, by performing normalization on the vectors, we obtain soft class-wise distributions for source and target domains.

\footnotetext{
Algorithm 1: Learning class-aware distribution alignment between source and target data.
}

(1) Produce C-dimensional segmentation score maps $F\left(x_{s}\right)$ and $F\left(x_{t}\right)$ for each example.

(2) Convert the segmentation score maps to prediction probability maps $Q_{x_{s}}$ and $Q_{x_{t}}$ using softmax.

(3) Compute C-dimensional soft count vectors $\left[P_{x_{s}}^{c}\right]_{c}$ and $\left[P_{x_{t}}^{c}\right]_{c}$ by adding the class prediction probabilities in each pixel. That is: $P_{x_{s}}^{c}=$ $\sum_{i=1}^{N} Q_{x_{s}}^{(i, c)}$ and $P_{x_{t}}^{c}=\sum_{i=1}^{N} Q_{x_{t}}^{(i, c)}$.

(4) Normalize the soft count vectors $\left[P_{x_{s}}^{c}\right]_{c}$ and $\left[P_{x_{t}}^{c}\right]_{c}$ by dividing each element by the sum of elements in the vector, thus producing the class-wise data distributions $\hat{P}_{x_{s}}$ and $\hat{P}_{x_{t}}$.

(5) Minimize the loss $\mathcal{L}_{\text {dist }}\left(x_{s}, x_{t}\right)$ in equation 4 through backpropagation.

In this algorithm we first estimate the class distribution in the source and target domains. After obtaining the soft class-wise distributions $\hat{P}_{x_{s}}$ and $\hat{P}_{x_{t}}$ for the source and target domains respectively, we compute the KL-divergence [22] loss to measure the class-wise 
distribution difference between the source and target data, denoted by

$$
\mathcal{L}_{\text {dist }}\left(x_{s}, x_{t}\right)=-\frac{1}{C} \sum_{c=1}^{C} \hat{P}_{x_{s}}^{(c)} \log \frac{\hat{P}_{x_{s}}^{(c)}}{\hat{P}_{x_{t}}^{(c)}}
$$

We add this loss term to the combined network loss and so encourage the source and target distributions to be similar.

\subsection{Objective Function for UDA in Semantic Segmentation}

During training, the model is learned by jointly minimizing the loss terms described above. The overall loss function for UDA in semantic segmentation is given by:

$$
\begin{aligned}
\mathcal{L}=\frac{1}{\left|D_{s}\right|} \sum_{x_{s}} \mathcal{L}_{\text {seg }}\left(x_{s}, y_{s}\right)+\frac{\lambda_{1}}{\left|D_{t}\right|} & \sum_{x_{t}} \mathcal{L}_{\text {ent }}\left(x_{t}\right)+ \\
& \frac{\lambda_{2}}{|D|} \sum_{\left(x_{s}, x_{t}\right)} \mathcal{L}_{\text {dist }}\left(x_{s}, x_{t}\right)
\end{aligned}
$$

where $|D|=\min \left(\left|D_{s}\right|,\left|D_{t}\right|\right)$ to account for the possibility of different number of examples in the source and target sets, and $\lambda_{1}, \lambda_{2}$ control the tradeoff among the three loss terms.

\subsection{Network Architecture and Training}

In this section, we combine the components described above. The network architecture is illustrated in Figure 1.

Segmentation Network Architecture. To obtain high-quality segmentation results, it is critical to choose a strong baseline model. In this work, we use Deeplab-V2 [4] with a pretrained ResNet-101 [16] model as the base semantic segmentation network $F$. Similar to several recent works on UDA $[39,41]$, we remove the multi-scale fusion strategy due to limited memory, and remove the last classification layer which is not needed in our problem. To better capture the scene context, we apply Atrous Spatial Pyramid Pooling (ASPP) [4] as the final classifier after the last layer. Following the setting in [4], the sampling rates are set to $\{6,12,18,24\}$. We also change the strides of the last layers conv 4 and conv 5 using dilated convolution layers to enlarge the receptive field. Finally, an up-sampling layer is applied to match the size of the input. The segmentation outputs of source and target inputs are passed to the three loss terms.

Network Training. To train the model, we first process the source input to optimize the segmentation network using $\mathcal{L}_{\text {seg }}$ and output the segmentation prediction $Q_{x_{s}}$. Likewise, we generate the output $Q_{x_{t}}$ for the target input, and pass it to optimize $\mathcal{L}_{\text {ent }}$. Finally, after calculating the soft counts per class for $P_{x_{s}}$ along with $P_{x_{t}}$, we obtain $\hat{P}_{x_{s}}$ and $\hat{P}_{x_{t}}$ which are passed to optimize $\mathcal{L}_{\text {dist }}$. We use the Pytorch deep learning framework [32] to implement our network on two NVIDIA-SMI GPU with 16 GB memory in total. We use a Stochastic Gradient Descent [3] optimizer with momentum of 0.9 and a weight decay of $10^{-4}$ to train the model. The initial learning rate is set to 0.001 and decayed using the polynomial decay schedule of [16]. The input size for the source and target domains is given by $512 \times 512$, and the batch size is set as 2 . The two loss weight factors $\lambda_{1}$ and $\lambda_{2}$ are set to 0.001 and 0.1 , respectively.
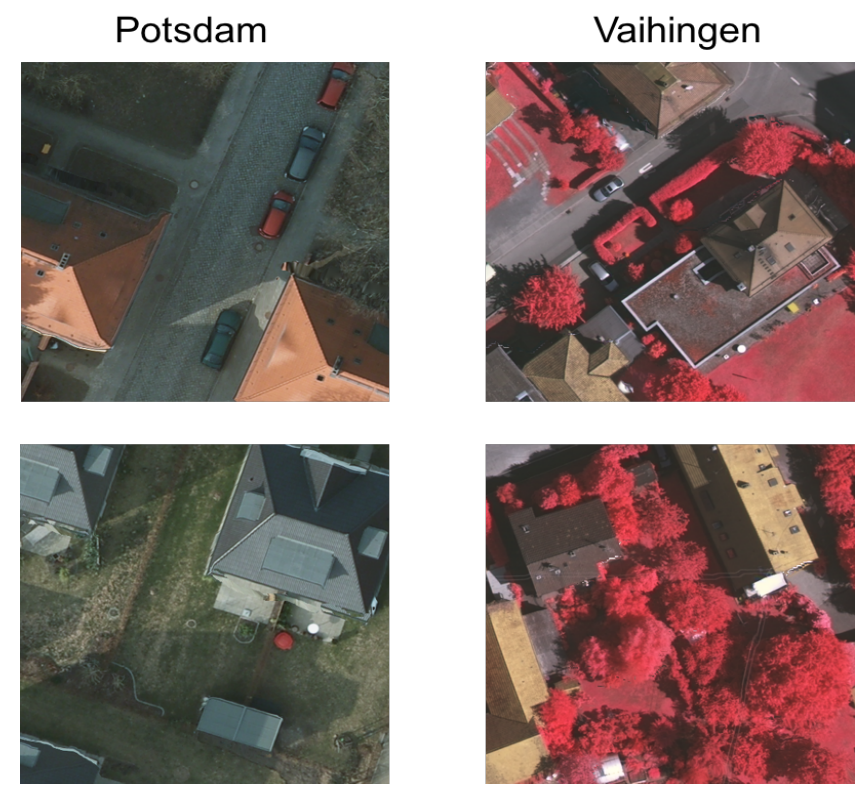

Figure 2: Example images from the Potsdam and Vaihingen datasets.

\section{EXPERIMENTS}

In this section, we present experimental results using several evaluation metrics. We evaluate the proposed approach and compare it to known approaches using standard evaluation datasets.

\subsection{Datasets}

To evaluate the proposed method, we follow a common evaluation procedure as described in $[1,2]$. We use the ISPRS (WGII/4) 2D semantic segmentation benchmark dataset [23], which is part of the ISPRS 2D semantic labeling challenge. To strengthen this evaluation procedure we add an additional task of adapting from the target set into the source set (in addition to adapting from the source set to the target set).

We are specifically interested in aerial image segmentation with its unique challenges stemming from appearance differences due to sensors, seasonal changes, illumination changes, and other natural phenomena, and which are represented well in this dataset. Following the standard evaluation procedure, we use two cities, Potsdam and Vaihingen, as our source and target domains respectively. Each pixel in each image is annotated with one of six classes of ground truth objects: building, tree, low vegetation, car, clutter/background, and impervious surfaces. The train/val/test sets is detailed in in [2]. Besides, we also conduct an additional evaluation where we reverse their roles. This in essence doubles our evaluation set.

We use images from the original digital surface model (DSM) for our domain adaptation task. The pixel density in these datasets, while high, does not match. Potsdam has a pixel density of $5 \mathrm{~cm}$ per pixel whereas Vaihingen has a pixel density of $7 \mathrm{~cm}$ per pixel. Such difference contributes to one aspect of domain shift between the Potsdam source and Vaihingen target datasets. As stated in [1], 
Table 1: Evaluation results using the Vaihingen target domain. The symbol $*$ indicates data not provided in reference paper. DBDA is the proposed approach and DBDA ${ }^{\{1,2\}}$ are variants of the proposed approach provided for the ablation study.

\begin{tabular}{|l|l||ccccc|}
\hline Category & Method & Average Accuracy & Precision & Recall & F1-score & IoU \\
\hline Baseline & Source-only & 0.471 & 0.414 & 0.471 & 0.316 & 0.214 \\
Approaches & FCNs in wild [19] & 0.486 & $*$ & $*$ & $*$ & 0.309 \\
& AUDA [2] & 0.520 & 0.540 & 0.520 & 0.490 & 0.300 \\
& DUDA [1] & 0.588 & $*$ & $*$ & $*$ & 0.349 \\
& AdvEnt [41] & 0.308 & 0.333 & 0.308 & 0.202 & 0.129 \\
\hline Proposed & DBDA $^{1}$ & 0.329 & 0.383 & 0.329 & 0.236 & 0.237 \\
approach & DBDA $^{2}$ & 0.554 & 0.546 & 0.554 & 0.510 & 0.367 \\
& DBDA & $\mathbf{0 . 5 9 1}$ & $\mathbf{0 . 5 6 8}$ & $\mathbf{0 . 5 9 1}$ & $\mathbf{0 . 5 3 9}$ & $\mathbf{0 . 3 9 3}$ \\
\hline
\end{tabular}

Table 2: Evaluation results when reversing the roles of source and target domains (using Potsdam as target). Fewer baseline methods are reported compared with Table 1 because some of the compared methods do not report this reverse evaluation. DBDA is the proposed approach and DBDA ${ }^{\{1,2\}}$ are variants of the proposed approach provided for the ablation study.

\begin{tabular}{|l|l||ccccc|}
\hline Category & Method & Average Accuracy & Precision & Recall & F1-score & IoU \\
\hline Baseline & Source-only & 0.396 & 0.512 & 0.396 & 0.372 & 0.243 \\
approaches & DUDA [1] & 0.363 & 0.365 & 0.363 & 0.318 & 0.309 \\
& AdvEnt [41] & 0.369 & 0.548 & 0.369 & 0.382 & 0.268 \\
\hline Proposed & DBDA $^{1}$ & 0.362 & 0.436 & 0.362 & 0.362 & 0.246 \\
approach & DBDA $^{2}$ & 0.451 & 0.582 & 0.451 & 0.479 & 0.319 \\
& DBDA & $\mathbf{0 . 4 5 5}$ & $\mathbf{0 . 5 8 3}$ & $\mathbf{0 . 4 5 5}$ & $\mathbf{0 . 4 8 5}$ & $\mathbf{0 . 3 3 0}$ \\
\hline
\end{tabular}

Table 3: IoU evaluation results for every class on the Vaihingen target domain. Fewer baseline methods are reported compared with Table 3 because some of the compared methods do not report this reverse evaluation.

\begin{tabular}{|l|l||ccccccc|}
\hline Category & Method & Imp.Sur. & Bui. & Lo. Veg. & Tree & Car & Clu.Backgr. & mIoU \\
\hline Baseline & Source-only & 0.413 & 0.074 & 0.022 & 0.306 & 0.019 & 0.451 & 0.214 \\
approaches & AdvEnt [41] & 0.414 & 0.024 & 0.039 & 0.013 & 0.232 & 0.052 & 0.129 \\
\hline Proposed approach & DBDA & $\mathbf{0 . 5 6 0}$ & $\mathbf{0 . 1 0 6}$ & $\mathbf{0 . 3 1 2}$ & $\mathbf{0 . 5 2 8}$ & $\mathbf{0 . 2 6 6}$ & $\mathbf{0 . 5 8 9}$ & $\mathbf{0 . 3 9 3}$ \\
\hline
\end{tabular}

this set has two more domain shift factors having to do with sensor variation and variation of class representations.

Each pixel in each image is annotated with one of six classes of ground truth objects: building, tree, low vegetation, car, clutter/background, and impervious surfaces. The Potsdam dataset contains 38 TOP images of size $6000 \times 6000$, whereas the Vaihingen dataset contains 33 TOP images with the same size. Following the split criterion in [1,2], each image is divided into several squares of size 512 by 512 for both training and testing. 32 images of the Potsdam dataset are used for training and the remaining six are used for testing. In the Vaihingen dataset 27 out of 33 images are used for training and the remaining 6 for testing. Several examples from the source domain (Potsdam) and the target domain (Vaihingen) are shown in Figure 2.

\subsection{Evaluation Metrics}

To evaluate the proposed method, we use five commonly used metrics following baseline methods $[1,2]$ : accuracy, recall, precision, F1-score, and Intersection over Union (IoU). The metrics are calculated with true positive (TP), false positive (FP), true negative (TN), and false negative (FN). For semantic segmentation, TP and
TN measure the number of pixels correctly predicted, whereas FP and FN measure the number of pixels incorrectly predicted. Since we have six different classes, the metrics are calculated as the mean value over the values calculated for all classes separately. IoU is computed as follows:

$$
I o U=\frac{T P}{T P+F P+F N}
$$

\subsection{Results}

In this section, we report experimental results of our proposed distribution-based domain adaptation approach (DBDA) and compare it with different baseline approaches. The baseline methods include: Source-only, a method trained on only the source domain using the backbone segmentation network of Deeplab-V2; FCNs in wild [19], a domain adaptation method for semantic segmentation; AUDA [2], an unsupervised domain adaptation approach for semantic segmentation of aerial images; DUDA [1], another domain adaptation algorithm for semantic segmentation of aerial images; AdvEnt [41], an adversarial domain adaptation approach which directly minimizes the entropy between the target and source domains. We show that the proposed DBDA approach improves on 
state-of-the-art performance when evaluated on the ISPRS aerial benchmark.

In addition, we perform a ablation study of our proposed DBDA network termed DBDA ${ }^{1}$ and $\mathrm{DBDA}^{2}$ in which we remove the classaware distribution alignment loss and the entropy minimization loss, respectively. Among these methods, FCNs in wild, AUDA, AdvEnt, and DUDA are all adversarial learning methods.

Table 1 presents the evaluation results of semantic segmentation on the Vaihingen test data in terms of the five measures: mean accuracy, precision, recall, F1-score and IoU. As can be observed, our proposed DBDA ${ }^{2}$ approach, obtains better performance compared with the baseline approaches in almost all measures. It validates the advantage in learning the class-wise distribution difference between the source and target domains, thus addresseing the domain shift between the two domains. By adding the entropy minimization component to the proposed approach we observe that the proposed DBDA approach surpasses all other methods. This demonstrates that our proposed method addresses the issue of domain shift and guarantees high prediction certainty on target data. In addition, our method does not involve any adversarial training as used for image translation in AUDA [2] and DUDA [1], and so is easier to train.

Note that when using minimum entropy alone (DBDA1), the performance is much lower compared with the proposed DBDA2 and DBDA approaches. The poor performance of AdvEnt on the test set demonstrates that using adversarial training to adapt domain shift may not work well on aerial data where there are large difference of appearance between the different domains. The poor performance of MinEnt on the test set indicates that domain discrepancy remains high even though the prediction certainty is maximized in the target domain. To evaluate the contribution of class-wise distribution alignment, we perform an ablation study as shown in the DBDA $\dagger$ entry in Table 1.

To further demonstrate the effectiveness of our method, we perform an additional evaluation where we reverse the role of the source and target domains. In this additional evaluation we use Vaihingen as source and Potsdam as target. Table 2 shows the evaluation performance. As can be observed in the table, the proposed DBDA approach outperforms the baseline methods in all metrics. This demonstrates again that the proposed approach effectively addresses domain shift between the two domains. We also observe that AdvEnt and DBDA ${ }^{1}$ show better performance than DUDA for most metrics, which validates the usage of entropy minimization to achieve high prediction certainty in the target domain.

We also investigate the effect of the proposed method on every class separately. Given that IoU is the most common measure used to evaluate semantic segmentation, we measure per class IoU performance of the proposed approach and the compared methods. The results are provided in Table 3. As reported in Table 3, our method increases the accuracy of objects which are hard to detect with high margin by comparing to the baselines, e.g.tree and car. In addition, the objects like tree and building are mostly related to sensor type [2], so our method achieves better performance than most existing approaches regarding the domain shift of sensor type, which demonstrates that our DBDA is able to reduce domain shift between the two domains, thus improving on the known state-ofthe-art in this area.

\section{CONCLUSION}

In this paper, we address the problem of domain adaptation for semantic segmentation of aerial images using entropy minimization and class-wise distribution alignment. Our approach improves on state-of-the-art performance, and is shown to be efficient in addressing domain shift on a challenging aerial dataset. We demonstrate the benefit of applying soft class-wise distribution alignment which results in improved performance compared with existing work. The combination of class-wise distribution alignment and entropy minimization further improves the performance of the proposed approach. In future work, we plan to extend this work to include semi-supervised learning scenarios where a small set of labeled data is available.

\section{REFERENCES}

[1] Bilel Benjdira, Adel Ammar, Anis Koubaa, and Kais Ouni. 2020. Data-Efficient Domain Adaptation for Semantic Segmentation of Aerial Imagery Using Generative Adversarial Networks. Applied Sciences 10, 3 (2020), 1092.

[2] Bilel Benjdira, Yakoub Bazi, Anis Koubaa, and Kais Ouni. 2019. Unsupervised domain adaptation using generative adversarial networks for semantic segmentation of aerial images. Remote Sensing 11, 11 (2019), 1369.

[3] Léon Bottou. 2010. Large-scale machine learning with stochastic gradient descent. In Proceedings of COMPSTAT'2010. Springer, Paris, France, 177-186.

[4] Liang-Chieh Chen, George Papandreou, Iasonas Kokkinos, Kevin Murphy, and Alan L Yuille. 2017. Deeplab: Semantic image segmentation with deep convolutional nets, atrous convolution, and fully connected crfs. IEEE transactions on pattern analysis and machine intelligence 40, 4 (2017), 834-848.

[5] Minghao Chen, Hongyang Xue, and Deng Cai. 2019. Domain adaptation for semantic segmentation with maximum squares loss. In Proceedings of the IEEE International Conference on Computer Vision. IEEE, Long Beach, CA, 2090-2099.

[6] Yuhua Chen, Wen Li, and Luc Van Gool. 2018. Road: Reality oriented adaptation for semantic segmentation of urban scenes. In Proceedings of the IEEE Conference on Computer Vision and Pattern Recognition. IEEE, Salt Lake City, UT, 7892-7901.

[7] Ying Chen, Xu Ouyang, and Gady Agam. 2019. ChangeNet: Learning to detect changes in satellite images. In Proceedings of the 3rd ACM SIGSPATIAL International Workshop on AI for Geographic Knowledge Discovery. Association for Computing Machinery, Chicago,IL, 24-31.

[8] Yi-Hsin Chen, Wei-Yu Chen, Yu-Ting Chen, Bo-Cheng Tsai, Yu-Chiang Frank Wang, and Min Sun. 2017. No more discrimination: Cross city adaptation of road scene segmenters. In Proceedings of the IEEE International Conference on Computer Vision. IEEE, Venice, Italy, 1992-2001.

[9] Ziliang Chen, Jingyu Zhuang, Xiaodan Liang, and Liang Lin. 2019. Blending-target domain adaptation by adversarial meta-adaptation networks. In Proceedings of the IEEE/CVF Conference on Computer Vision and Pattern Recognition. IEEE, Long Beach Convention and Entertainment Center, 2248-2257.

[10] Marius Cordts, Mohamed Omran, Sebastian Ramos, Timo Rehfeld, Markus Enzweiler, Rodrigo Benenson, Uwe Franke, Stefan Roth, and Bernt Schiele. 2016. The cityscapes dataset for semantic urban scene understanding. In Proceedings of the IEEE conference on computer vision and pattern recognition. IEEE, Caesars Palace, Las Vegas, NV, 3213-3223.

[11] Jurgen Everaerts et al. 2008. The use of unmanned aerial vehicles (UAVs) for remote sensing and mapping. The International Archives of the Photogrammetry, Remote Sensing and Spatial Information Sciences 37, 2008 (2008), 1187-1192.

[12] Mark Everingham, SM Ali Eslami, Luc Van Gool, Christopher KI Williams, John Winn, and Andrew Zisserman. 2015. The pascal visual object classes challenge: A retrospective. International journal of computer vision 111, 1 (2015), 98-136.

[13] Chelsea Finn, Pieter Abbeel, and Sergey Levine. 2017. Model-agnostic metalearning for fast adaptation of deep networks. In International Conference on Machine Learning. PMLR, PMLR, 1126-1135.

[14] Rui Gong, Yuhua Chen, Danda Pani Paudel, Yawei Li, Ajad Chhatkuli, Wen Li, Dengxin Dai, and Luc Van Gool. 2021. Cluster, Split, Fuse, and Update: MetaLearning for Open Compound Domain Adaptive Semantic Segmentation. In Proceedings of the IEEE/CVF Conference on Computer Vision and Pattern Recognition. IEEE, Nashville, 8344-8354.

[15] Yves Grandvalet and Yoshua Bengio. 2005. Semi-supervised learning by entropy minimization. In Advances in neural information processing systems. Vancouver, British Columbia, Canada, 529-536.

[16] Kaiming He, Xiangyu Zhang, Shaoqing Ren, and Jian Sun. 2016. Deep residual learning for image recognition. In Proceedings of the IEEE conference on computer vision and pattern recognition. Caesars Palace, Las Vegas, NV, 770-778.

[17] Sepp Hochreiter, A Steven Younger, and Peter R Conwell. 2001. Learning to learn using gradient descent. In International Conference on Artificial Neural Networks. 
Springer, Springer, 87-94

[18] Judy Hoffman, Eric Tzeng, Taesung Park, Jun-Yan Zhu, Phillip Isola, Kate Saenko, Alexei Efros, and Trevor Darrell. 2018. Cycada: Cycle-consistent adversarial domain adaptation. In International conference on machine learning. Omnipress, Stockholmsmassan, Stockholm, Sweden, 1989-1998.

[19] Judy Hoffman, Dequan Wang, Fisher Yu, and Trevor Darrell. 2016. Fcns in the wild Pixel-level adversarial and constraint-based adaptation. CoRR, abs/1612.02649 (2016).

[20] Seunghoon Hong, Hyeonwoo Noh, and Bohyung Han. 2015. Decoupled deep neural network for semi-supervised semantic segmentation. In Advances in neural information processing systems. Palais des Congres de Montreal, Montreal CANADA, 1495-1503.

[21] Weixiang Hong, Zhenzhen Wang, Ming Yang, and Junsong Yuan. 2018. Con ditional generative adversarial network for structured domain adaptation. In Proceedings of the IEEE Conference on Computer Vision and Pattern Recognition. IEEE, Salt Lake City, UT, 1335-1344.

[22] Solomon Kullback and Richard A Leibler. 1951. On information and sufficiency. The annals of mathematical statistics 22, 1 (1951), 79-86.

[23] S Labeling and B Vaihingen. 2016. Use of the Stair Vision Library within the ISPRS Use of the Stair Vision Library within the ISPRS 2D. (2016).

[24] Fahad Lateef and Yassine Ruichek. 2019. Survey on semantic segmentation using deep learning techniques. Neurocomputing 338 (2019), 321-348.

[25] Da Li and Timothy Hospedales. 2020. Online meta-learning for multi-source and semi-supervised domain adaptation. In European Conference on Computer Vision Springer, Springer, Scottish Event Campus, 382-403.

[26] Yunsheng Li, Lu Yuan, and Nuno Vasconcelos. 2019. Bidirectional learning for domain adaptation of semantic segmentation. In Proceedings of the IEEE Conference on Computer Vision and Pattern Recognition. IEEE, Long Beach, CA, 6936-6945.

[27] Shuang Liu, Olivier Bousquet, and Kamalika Chaudhuri. 2017. Approximation and convergence properties of generative adversarial learning. In Advances in Neural Information Processing Systems. Morgan Kaufmann Publishers Inc, Long Beach Convention Center, Long Beach, 5545-5553.

[28] Jonathan Long, Evan Shelhamer, and Trevor Darrell. 2015. Fully convolutional networks for semantic segmentation. In Proceedings of the IEEE conference on computer vision and pattern recognition. IEEE, Boston, MA, USA, 3431-3440.

[29] Mingsheng Long, Yue Cao, Jianmin Wang, and Michael Jordan. 2015. Learning transferable features with deep adaptation networks. In International conference on machine learning. Omnipress, Lille, France, 97-105.

[30] Mingsheng Long, Han Zhu, Jianmin Wang, and Michael I Jordan. 2016. Unsupervised domain adaptation with residual transfer networks. In Advances in neural information processing systems. Morgan Kaufmann Publishers Inc, International Barcelona Convention Center, Barcelona, Spain, 136-144.

[31] Mingsheng Long, Han Zhu, Jianmin Wang, and Michael I Jordan. 2017. Deep transfer learning with joint adaptation networks. In Proceedings of the 34th International Conference on Machine Learning-Volume 70. JMLR. org, 2208-2217.

[32] Adam Paszke, Sam Gross, Soumith Chintala, Gregory Chanan, Edward Yang Zachary DeVito, Zeming Lin, Alban Desmaison, Luca Antiga, and Adam Lerer. 2017. Automatic differentiation in pytorch. NIPS Workshop (2017).

[33] Stephan R Richter, Vibhav Vineet, Stefan Roth, and Vladlen Koltun. 2016. Playing for data: Ground truth from computer games. In European conference on computer vision. Springer, Amsterdam, The Netherlands, 102-118.

[34] German Ros, Laura Sellart, Joanna Materzynska, David Vazquez, and Antonio M Lopez. 2016. The synthia dataset: A large collection of synthetic images for semantic segmentation of urban scenes. In Proceedings of the IEEE conference on computer vision and pattern recognition. IEEE, Caesars Palace, Las Vegas, NV, 3234-3243.

[35] Claude E Shannon. 1948. A mathematical theory of communication. Bell system technical journal 27, 3 (1948), 379-423.

[36] Kihyuk Sohn, Sifei Liu, Guangyu Zhong, Xiang Yu, Ming-Hsuan Yang, and Manmohan Chandraker. 2017. Unsupervised domain adaptation for face recognition in unlabeled videos. In Proceedings of the IEEE International Conference on Computer Vision. Venice, Italy, 3210-3218.

[37] Nasim Souly, Concetto Spampinato, and Mubarak Shah. 2017. Semi supervised semantic segmentation using generative adversarial network. In Proceedings of the IEEE International Conference on Computer Vision. IEEE, Venice, Italy, 5688-5696.

[38] Jost Tobias Springenberg. 2015. Unsupervised and semi-supervised learning with categorical generative adversarial networks. ICLR (2015).

[39] Yi-Hsuan Tsai, Wei-Chih Hung, Samuel Schulter, Kihyuk Sohn, Ming-Hsuan Yang, and Manmohan Chandraker. 2018. Learning to adapt structured output space for semantic segmentation. In Proceedings of the IEEE Conference on Computer Vision and Pattern Recognition. IEEE, Salt Lake City, UT, 7472-7481.

[40] Eric Tzeng, Judy Hoffman, Kate Saenko, and Trevor Darrell. 2017. Adversarial discriminative domain adaptation. In Proceedings of the IEEE conference on computer vision and pattern recognition. IEEE, Hawaii Convention Center, Honolulu, HI, 7167-7176.

[41] Tuan-Hung Vu, Himalaya Jain, Maxime Bucher, Matthieu Cord, and Patrick Pérez. 2019. Advent: Adversarial entropy minimization for domain adaptation in semantic segmentation. In Proceedings of the IEEE Conference on Computer Vision and Pattern Recognition. IEEE, Long Beach, CA, 2517-2526.

[42] Yunchao Wei, Huaxin Xiao, Honghui Shi, Zequn Jie, Jiashi Feng, and Thomas S Huang. 2018. Revisiting dilated convolution: A simple approach for weakly-and semi-supervised semantic segmentation. In Proceedings of the IEEE Conference on Computer Vision and Pattern Recognition. IEEE, Salt Lake City, UT, 7268-7277.

[43] Zuxuan Wu, Xintong Han, Yen-Liang Lin, Mustafa Gokhan Uzunbas, Tom Goldstein, Ser Nam Lim, and Larry S Davis. 2018. Dcan: Dual channel-wise alignment networks for unsupervised scene adaptation. In Proceedings of the European Conference on Computer Vision (ECCV). Springer Science and Business Media, Munich, Germany, 518-534.

[44] Shaoan Xie, Zibin Zheng, Liang Chen, and Chuan Chen. 2018. Learning semantic representations for unsupervised domain adaptation. In International Conference on Machine Learning. Omnipress, StockholmsmÃd’ssan, Stockholm, Sweden, 54235432.

[45] Hongliang Yan, Yukang Ding, Peihua Li, Qilong Wang, Yong Xu, and Wangmeng Zuo. 2017. Mind the class weight bias: Weighted maximum mean discrepancy for unsupervised domain adaptation. In Proceedings of the IEEE Conference on Computer Vision and Pattern Recognition. IEEE, Hawai Convention Center, Honolulu, HI, 2272-2281

[46] Jian Zhang, Lei Qi, Yinghuan Shi, and Yang Gao. 2020. Generalizable semantic segmentation via model-agnostic learning and target-specific normalization. arXiv preprint arXiv:2003.12296 (2020).

[47] Jun-Yan Zhu, Taesung Park, Phillip Isola, and Alexei A Efros. 2017. Unpaired image-to-image translation using cycle-consistent adversarial networks. In Proceedings of the IEEE international conference on computer vision. IEEE, Venice, Italy, 2223-2232.

[48] Yang Zou, Zhiding Yu, BVK Vijaya Kumar, and Jinsong Wang. 2018. Unsupervised domain adaptation for semantic segmentation via class-balanced self-training. In Proceedings of the European conference on computer vision (ECCV). Springer Science and Business Media, Munich, Germany, 289-3,05. 